\title{
DEMICONTINUITY, HEMICONTINUITY AND MONOTONICITY
}

\author{
BY TOSIO KATO \\ Communicated by F. Browder, March 12, 1964
}

Recently the notions of monotone, demicontinuous and hemicontinuous functions have been introduced in connection with nonlinear problems in functional analysis (Browder $[1 ; 2 ; 3 ; 4 ; 5]$, Minty $[6 ; 7 ; 8])$. The object of the present note is to show that under rather general conditions, hemicontinuity is equivalent to demicontinuity for monotone functions.

Let $X$ be a (real or complex) Banach space and $X^{*}$ its adjoint space as the set of all bounded conjugate-linear functionals on $X$. The value of $f \in X^{*}$ at $u \in X$ is denoted by $(f, u)$. We use the notations $\rightarrow$ and $\rightarrow$ for strong convergence in $X$ (or in $X^{*}$ or in the set of real numbers) and weak* convergence in $X^{*}$, respectively.

Let $G$ be a function from $X$ to $X^{*}$ with domain $D=D(G) \subset X$. $G$ is said to be demicontinuous if $u_{n} \in D, n=1,2,3, \cdots, u \in D$ and $u_{n} \rightarrow u$ imply $G u_{n} \rightarrow G u$. $G$ is hemicontinuous if $u \in D, v \in X$ and $u+t_{n} v \in D$, where $t_{n}$ is a sequence of positive numbers such that $t_{n} \rightarrow 0$, imply $G\left(u+t_{n} v\right) \rightarrow G u$. We shall say that $G$ is locally bounded if $u_{n} \in D, u \in D$ and $u_{n} \rightarrow u$ imply that $G u_{n}$ is bounded. Obviously a demicontinuous function is hemicontinuous and locally bounded.

$G$ is said to be monotone if $\operatorname{Re}(G u-G v, u-v) \geqq 0$ for $u, v \in D$.

These definitions may be void if $D$ is too arbitrary. In what follows we shall assume that $D$ is quasi-dense. By this we mean that for each $u \in D$ there is a dense subset $M_{u}$ of $X$ such that for each $v \in M_{u}$, $u+t v \in D$ for sufficiently small $t>0$ (the smallness of $t$ depending on $v)$. Thus any open subset of $X$ as well as any dense linear manifold of $X$ is quasi-dense.

Theorem 1. Let $G$ be a monotone function from $X$ to $X^{*}$ with a quasidense domain $D$. Then $G$ is demicontinuous if and only if it is hemicontinuous and locally bounded.

Proof. By the remark given above, it suffices to prove the "if" part. Suppose $G$ is hemicontinuous and locally bounded. Let $u_{n} \rightarrow u$, $u_{n}, u \in D$. We have to show that $G u_{n} \rightarrow G u$. Obviously we may assume that $u_{n} \neq u$.

Let $M_{u}$ be the dense subset of $X$ used in the definition of $D$ being quasi-dense. Let $v \in M_{u}$ and $t_{n}=\left\|u_{n}-u\right\|^{1 / 2}$. Then $t_{n}>0, t_{n} \rightarrow 0$, $w_{n}=u+t_{n} v \in D$ for sufficiently large $n$ and 


$$
G w_{n} \rightarrow G u \text {. }
$$

Now the monotonicity of $G$ implies

(2) $0 \leqq \operatorname{Re}\left(G u_{n}-G w_{n}, u_{n}-w_{n}\right)=\operatorname{Re}\left(G u_{n}-G w_{n}, u_{n}-u-t_{n} v\right)$.

$G u_{n}$ is bounded since $G$ is locally bounded. $G w_{n}$ is bounded by (1). Hence

$$
t_{n}^{-1} \operatorname{Re}\left(G u_{n}-G w_{n}, u_{n}-u\right) \rightarrow 0
$$

because $\left\|t_{n}^{-1}\left(u_{n}-u\right)\right\|=t_{n} \rightarrow 0$. Also $\left(G w_{n}, v\right) \rightarrow(G u, v)$ by (1). Dividing (2) by $t_{n}$ and letting $n \rightarrow \infty$, we thus obtain

$$
\lim \inf \operatorname{Re}\left(G u_{n}-G u,-v\right) \geqq 0 .
$$

(3) is true for any $v \in M_{u}$. Since $M_{u}$ is dense in $X$ and $G u_{n}$ is bounded in $X^{*}$, it follows that (3) is true for every $v \in X$. Replacing $v$ by $-v$ (and also by $\pm i v$ if $X$ is complex) and putting the results together, we obtain

$$
\lim \left(G u_{n}-G u, v\right)=0, \quad v \in X .
$$

This proves that $G u_{n} \rightarrow G u$, q.e.d.

REMARK 1. Theorem 1 shows that a monotone hemicontinuous function that maps bounded sets into bounded sets is a notion stronger than a monotone demicontinuous function. (Such functions are considered in [2-III] and [5].)

REMARK 2. It is not clear whether the assumption of local boundedness in Theorem 1 can be eliminated. But this is the case if $X$ is finite-dimensional. We have namely

Theorem 2. Let $X$ be a finite-dimensional Banach space. Let $G$ be a monotone function from $X$ to $X^{*}$ with a quasi-dense domain $D$. Then $G$ is continuous if and only if it is hemicontinuous.

Proof. Since continuity and demicontinuity are equivalent when $X$ is finite-dimensional, it suffices to show that $G$ is locally bounded if it is hemicontinuous; then the result follows from Theorem 1.

Suppose that $G$ is hemicontinuous but not locally bounded. Then there is a $u \in D$ and a sequence $u_{n} \in D$ such that $u_{n} \rightarrow u$ but $G u_{n}$ is unbounded. We may assume without loss of generality that $\left\|G u_{n}\right\|$ $=s_{n} \rightarrow \infty$. Let $M_{u}$ be as above and let $v \in M_{u}$. Take a $t>0$ so small that $u+t v \in D$. Then by monotonicity

$$
\begin{aligned}
0 & \leqq s_{n}^{-1} \operatorname{Re}\left(G u_{n}-G(u+t v), u_{n}-u-t v\right) \\
& =\operatorname{Re}\left(s_{n}^{-1} G u_{n}-s_{n}^{-1} G(u+t v), u_{n}-u-t v\right) .
\end{aligned}
$$


Now $s_{n}^{-1} G(u+t v) \rightarrow 0, u_{n}-u \rightarrow 0$ and $s_{n}^{-1} G u_{n}$ is bounded. On dividing (4) by $t>0$ and letting $n \rightarrow \infty$, we thus obtain

$$
\lim \inf \operatorname{Re}\left(s_{n}^{-1} G u_{n},-v\right) \geqq 0 .
$$

As in the proof of Theorem 1 , this leads to the result that $s_{n}^{-1} G u_{n}$ $\rightarrow 0$. But this is a contradiction, for $\left\|s_{n}^{-1} G u_{n}\right\|=1$ and weak* convergence is equivalent to strong convergence.

\section{BIBLIOGRAPHY}

1. F. E. Browder, The solvability of non-linear functional equations, Duke Math. J. 30 (1963), 557-566.

2. - Variational boundary value problems for quasi-linear elliptic equations, Proc. Nat. Acad. Sci. U.S.A. 50 (1963), 31-37, 592-598, 794-798.

3. - Nonlinear elliptic boundary value problems, Bull. Amer. Math. Soc. 69 (1963), 862-874.

4. - Nonlinear parabolic boundary value problems of arbitrary order, Bull. Amer. Math. Soc. 69 (1963), 858-861.

5. - Non-linear equations of evolution, Ann. of Math. (to appear).

6. G. J. Minty, Monotone (nonlinear) operators in Hilbert space, Duke Math. J. 29 (1962), 341-346.

7. - Two theorems on nonlinear functional equations in Hilbert space, Bull. Amer. Math. Soc. 69 (1963), 691-692.

8. - On a "monotonicity" method for the solution of nonlinear equations in Banach spaces, Proc. Nat. Acad. Sci. U.S.A. 50 (1963), 1038-1041.

University of California, Berkeley 